\title{
INTERESSES E PERSONALIDADE: DIFERENÇAS ENTRE SÉRIE E SEXO DE JOVENS DO ENSINO MÉDIO
}

\author{
Silvia Godoy \\ Mestre em Psicologia pela Universidade São Francisco - Itatiba, SP. Doutoranda do Programa de \\ Pós-Graduação em Distúrbios do Desenvolvimento, Universidade Presbiteriana Mackenzie - São \\ Paulo, SP.
}

\begin{abstract}
Ana Paula Porto Noronha
Doutora em Psicologia, Profissão e Ciência, pela Pontifícia Universidade Católica de Campinas. Docente do Programa de Pós-Graduação Stricto Sensu em Psicologia da Universidade São Francisco. Bolsista Produtividade em Pesquisa do CNPq.
\end{abstract}

\section{Resumo}

A escolha da profissão ainda se apresenta como um desafio nos variados momentos da vida e, por isso, tem sido objeto de investigação para pesquisadores da área. Nesse estudo analisaram-se as diferenças de interesses e personalidade em função do sexo e da série em 122 estudantes do Ensino Médio de uma escola particular do interior paulista. A idade dos sujeitos variou de 14 a 17 anos, sendo 44,3\% do sexo masculino. Os resultados apontaram preferência masculina pela dimensão Ciências Exatas e feminina pelas dimensões Artes e Comunicação, Ciências Biológicas e da Saúde e Ciências Humanas e Sociais Aplicadas. Com relação à personalidade, as mulheres se destacaram nas características voltadas para sentimentos de compaixão, ternura e amor, enquanto os homens nas características de persuasão, sedução, comando e independência. Concluiu-se que a área de orientação profissional precisa dispor cada vez mais de conhecimento teórico e prático no contexto social, político e econômico.

Palavras-chave: orientação profissional, interesse profissional, personalidade.

\section{INTERESTS AND PERSONALITY: DIFFERENCE BETWEEN GRADE AND GENDER AMONG THE INTERMEDIARY SCHOOL STUDENTS}

\begin{abstract}
Choosing a professional career or occupation has been a great challenge in different stages of their life, reason why this theme has been a focused item to be investigated by the researchers engaged in this area. This study looked into the differences between interests and personality based on sex and school level of 122 High School students of a private high school located in São Paulo State. These participants had between 14 and 17 years old, 44.3\% male. The results showed male preference fell into Exact Sciences while the female preference was for Arts and Communication, and the following fields of Sciences: Biologic and Health and Agrarian Human and Social. Regarding the personality women emphasized themselves in characteristics related to feelings of compassion, tenderness and love while men in the characteristics of persuasion, seduction, command and independence. It concludes that the professional orientation area needs to have even more theoretical and practical knowledge in the social, politics and economical context.
\end{abstract}

Key words: vocational guidance, professional interests, personality. 


\title{
INTERESES Y PERSONALIDADES: LAS DIFERENCIAS ENTRE EL CATEGORÍA Y GÉNERO DE LOS JÓVENES ESTUDIANTES DE SECUNDÁRIA
}

\begin{abstract}
Resumen
La elección de la profesión todavía constituye un reto en diversas etapas de la vida y por lo tanto ha sido objeto de investigación para los investigadores. En este estudio se analizaron las diferencias en los intereses y la personalidad por género y categoría en 122 estudiantes de secundaria de un colegio privado de San Pablo. La edad de los sujetos oscilaban entre 14 y 17 años, el 44,3\% hombre. Los resultados indicaron una preferencia masculina por dimensiones de la Ciencia Exacta y femenina, y de la Comunicación y las Artes, Ciencias Biológicas y Salud y Ciencias Sociales Aplicadas. En cuanto a la personalidad, las mujeres se destacaron en las características destinadas a sentimientos de compasión, ternura y amor, mientras que los hombres sobre las características de la persuasión, la seducción, el control y la independencia. Se concluye que el ámbito de las necesidades de orientación profesional tiene un conocimiento cada vez teórica y práctica en los ámbitos social, político y económico.

Palabras clave: orientación profesional, interés profesional, personalidad.
\end{abstract}

\section{INTRODUÇÃO}

O processo de Orientação Profissional (OP) envolve uma série de atividades, quadros de referência, orientações teóricas, concepções filosóficas, científicas e técnicas de trabalho. Além disso, implica informação sobre profissões e descoberta de capacidades pessoais. Desta forma, o conhecimento de si mesmo, o conhecimento das profissões e a escolha propriamente dita são os principais focos da OP, possibilitando ao indivíduo rever conceitos sobre si mesmo, sobre o mundo e sobre a vida, com o intuito de tornar mais claros os possíveis caminhos profissionais (Bohoslavsky, 1993). Sob esse aspecto, Nunes e Noronha (2009) enfatizam ainda que a avaliação em Orientação Profissional relaciona-se com a análise de construtos como interesses, necessidades de trabalho, valores, maturidade e indecisão profissional.

De acordo com Melo-Silva, Noce e Andrade (2003), o momento da escolha da profissão requer, muitas vezes, a intervenção de profissionais especializados. A OP configura-se como o campo de atividades que dispõe de conhecimentos teóricos e práticos destinados a facilitar o processo de escolha profissional e elaboração de projetos futuros, sobretudo, do adolescente. A escolha integra também um conjunto de determinantes sociais, econômicos e culturais, além das características pessoais, dentre eles, o interesse profissional (Duarte, 2008; Leitão \& Miguel, 2004), objeto de investigação desse estudo.

A ênfase no estudo dos interesses vem ganhando destaque na avaliação do construto, assim como na construção de métodos e estratégias para sua 
mensuração. No que se refere ao tema, Mattiazzi (1977) relata que desde o início do século XX, especialmente a partir de 1910, o conceito tem ocupado um importante espaço dentro da Psicologia Vocacional. Entretanto, apesar dos diversos estudos que procuram discutir o assunto, não havia à época, uma teoria que explicasse, de forma sustentável, a definição de interesse. Leitão e Miguel (2004) corroboram a idéia ao assinalar que desde a década de 60, não tem sido constatada qualquer evolução na clarificação do construto.

Mesmo defendendo que não há exatamente uma teoria sobre os interesses, Mattiazzi (1977) considera o construto como um processo comportamental dinâmico, sendo um fator motivador que orienta a atenção e conduz a busca por atividades específicas, determinando sua duração e intensidade. Mais contemporaneamente, Savickas (1999) definiu o construto interesse como uma tendência para a satisfação de necessidades e valores pessoais, influenciando na prontidão de um indivíduo para responder a determinados estímulos ambientais. No entanto, entende-se que os interesses estão associados à forma como os indivíduos procuram, no ambiente, realizar as suas necessidades e atender aos seus valores.

Uma teoria bastante estudada por pesquisadores em diversos países é a de Holland (1997). A concepção teórica do autor, intitulada Modelo Hexagonal, propõe integrar os seis tipos de personalidade, a saber, Realista, Investigativo, Artístico, Social, Empreendedor e Convencional (RIASEC) com as áreas profissionais, na qual os interesses profissionais são compreendidos como padrões de gosto, aversão ou neutralidade frente às atividades profissionais. 0 tipo Realista (R) refere-se, segundo o autor, ao pouco sociável, ao uso da força física e à preferência por atividades com elementos concretos. O tipo Intelectual (I) é analítico, introvertido e crítico, preferindo atividades que envolvam razão e curiosidade. O Artístico (A), por sua vez, utiliza mais frequentemente os sentimentos e a criatividade para lidar com as situações cotidianas.

Por outro lado, o Social (S) costuma agir mais pela intuição, apresentando interesse por atividades assistenciais de cuidado e bem-estar do outro. Entusiasmo, impulsividade e extroversão são características do tipo Empreendedor (E). Ao lado delas, há preocupação com o poder, posição social e liderança. Por fim, o Convencional (C) tem preferência por atividades burocráticas como finanças, além do gosto por tarefas repetitivas. Para Holland (1997), os interesses podem ser compreendidos como a expressão da 
personalidade no contexto do trabalho, à medida que enfatiza a importância da coerência entre as características pessoais (interesses e personalidade) e o ambiente de trabalho. Assim, o autor pressupõe que a escolha esteja imbricada com as características de personalidade e as características da profissão.

De forma a explicitar as proposições citadas anteriormente, algumas investigações serão apresentadas por se relacionarem com o tema do presente estudo. Com vistas a avaliar as características relacionadas à tipologia de Holland com a força de trabalho americana entre as décadas de 1960 e 2000, Reardon, Bullock e Meyer (2005) analisaram um censo nacional que classifica as ocupações de acordo com o RIASEC. O tipo Realista foi associado a mais ocupações em todas as décadas, variando entre 43 e 50\%. O tipo Artístico figurou entre o menos freqüente no mesmo período, com variações entre 2 e $5 \%$. As profissões Investigativas e Artísticas apresentaram níveis mais elevados de complexidade cognitiva das tarefas e, por fim, as profissões Convencionais e Realistas foram as menos complexas.

Especialmente no tocante à década de 2000, ao analisar a questão do sexo associado às ocupações, observou-se uma estabilidade do Realista para os homens, sendo que, para as mulheres, o tipo Convencional foi o mais freqüente até a década de 1990, porém em 2000 o tipo Empreendedor se destacou. Os achados permitiram afirmar que os homens estiveram nas ocupações Realista e Empreendedora entre $75 \%$ e $85 \%$ no período total, enquanto as mulheres estiveram em ocupações mais variadas ao longo das décadas, como a Convencional, Realista e Social e, na década de 2000, também com uma maior participação no Empreendedor. No que diz respeito à faixa etária e sexo dos trabalhadores, os homens mais jovens (16-39 anos) ocuparam profissões Realistas (48\%) e Empreendedoras (29\%), enquanto as mulheres, da mesma faixa etária, assumiam cargos nos tipos Empreendedor (30\%), Convencional (26\%) e Social (22\%).

Carless (1999) estudou a relação entre interesse e personalidade associados a gênero, medidos pelos instrumentos Self-Directed Search Career Explorer (SDS) e o Inventário de Personalidade NEO-PI. Participaram da pesquisa 139 adultos trabalhadores, sendo 48 homens e 91 mulheres, com média de idade 29 anos, que variou de 18 a 59. Os achados indicaram correlações significativas em ambos os sexos, quais sejam, o traço Extroversão correlacionou-se com o tipo Social $(r=0,71 ; p<0,01$ e $r=0,45 ; p<0,01)$ e com o 
tipo Empreendedor $(r=0,69 ; p<0,01$ e $r=0,52 ; p<0,01)$ para homens e mulheres, respectivamente. O traço Abertura associou-se com os tipos Artístico $(r=0,52 ; p<0,01)$, Investigativo $(r=0,54 ; p<0,01)$ e Convencional $(r=0,29$; $\mathrm{p}<0,05)$ para mulheres. Por sua vez, o traço Realização correlacionou-se com o tipo Empreendedor $(r=0,40 ; p<0,01$ e $r=0,23 ; p<0,05)$ para homens e mulheres; o traço Neuroticismo apresentou associação negativa com o tipo Social $(r=-0,42 ; p<0,01)$ para homens e o traço Socialização apresentou correlação significativa com o tipo Convencional $(r=0,40 ; p<0,01)$ para homens. Em resumo, de acordo com o autor, essa investigação sugere que exista relação entre interesses vocacionais e personalidade, ao mesmo tempo em que revela diferenças entre sexos, o que pode vir a contribuir para decisões mais eficazes da carreira.

Em outro estudo, Almeida, Guisande, Soares e Saavedra (2006) investigaram a entrada e sucesso no Ensino Superior em Portugal de 1407 alunos da Universidade do Minho, sendo 59\% mulheres, com a média de idade de 18,4 anos. Para conhecer a origem sócio-cultural das suas famílias de origem, os autores os classificaram de acordo com o grau máximo de escolaridade dos pais, tendo três categorias, a saber, baixo (equivalente ao Ensino Fundamental, Ciclo I), médio (equivalente ao Ensino Fundamental, Ciclo II até Ensino Médio) e alto (pais com cursos de graduação). Quanto aos cursos, foram divididos em quatro grandes áreas, quais sejam, Ciências naturais (CN), econômicas (CE), sociais (CS) e Engenharias (E). No que se refere à distribuição por sexo, as CN, CE e CS tiveram maior participação feminina, em todas as classes, sendo que a menor freqüência nesses casos foi de 58,3\% para CE na origem sócio-cultural alta. Já na Engenharia houve uma predominância masculina em todas as três origens.

Participantes de um programa de orientação profissional foram estudados por Osborn e Reardon (2006). Realizou-se análise de variância (ANOVA) nos dados coletados em 98 alunos do Ensino Médio para verificar se existia diferença de média significativa entre os tipos do SDS. Os tipos mais pontuados foram Artístico (32\%) e Realista (24\%) para os meninos, e Social (73\%) e Artístico (32\%) para as meninas. A ANOVA revelou diferenças significativas para dois tipos: Realista $(p<0,001)$ e Social $(p<0,05)$. Vale ressaltar que as profissões mais escolhidas entre os estudantes foram professor, advogado, e cantor, para o gênero feminino e atleta profissional, advogado e médico para o gênero 
masculino. Para o sexo masculino os tipos mais pontuados foram o Realista e Convencional; enquanto para o sexo feminino, Artístico e Empreendedor. Os autores concluíram, dentre outros aspectos, que o SDS é um instrumento com boas características psicométricas para estudantes do Ensino Médio.

Williams e Subich (2006) avaliaram 350 universitários, sendo 59\% do sexo feminino, com idade média de 22 anos para homens e 24 para mulheres com o objetivo de verificar diferenças de interesse em função do gênero, de acordo com a tipologia de Holland. Para isso, os autores aplicaram o SDS e os resultados apresentaram diferenças significativas em função do gênero para os tipos Realista e Social $(p<0,001)$ e também para o Investigativo $(p<0,01)$. Especificamente, as mulheres apresentaram menos experiências nos campos Realista e Investigativo e os homens, menos experiências no tipo Social.

Nessa mesma direção, Santos e Melo-Silva (2005), com intuito de investigar diferenças nas preferências por cursos de graduação associadas a gênero, realizaram um levantamento da lista dos alunos matriculados nos cursos da Universidade de São Paulo (USP), no campus de Ribeirão Preto, no ano de 2003. Os resultados revelaram que os cursos de economia, contabilidade, administração, informática, biomédica e medicina são os que concentram mais homens, enquanto enfermagem, fonoaudiologia, terapia ocupacional, nutrição, farmácia, psicologia, pedagogia, fisioterapia e química predominam as mulheres. Entretanto, as autoras supõem que exista uma divisão das profissões orientada por estereótipos associados a gênero, uma vez que as mulheres procuram mais freqüentemente cursos ligados aos cuidados e à assistência. Os homens, por sua vez, preferem profissões que envolvam raciocínio, cálculo e aquelas reconhecidas como de maior prestígio social.

No que tange à investigação dos perfis de interesse de uma população específica, Balbinotti, Valentini e Cândido (2006) estudaram os interesses profissionais de soldados do Exército Brasileiro por meio de um instrumento baseado na teoria de Holland (1963). A pesquisa contou com uma amostra de 352 soldados, com idades entre 18 e 32 anos, oriundos de várias localidades do estado do Rio Grande do Sul. Os resultados revelaram, inicialmente, que a variável faixa etária não determinou diferenças nos níveis de interesses profissionais dos sujeitos avaliados quanto às suas personalidades Realista, Investigativa, Artística, Empreendedora e Convencional. Desta forma, os achados indicaram diferenças significativas de média em função da faixa etária apenas 
para o tipo Social, sendo que os mais velhos obtiveram médias mais elevadas quanto às preferências por atividades que envolvam relacionamento com outras pessoas no intuito de ajudar, servir, educar e informar.

O estudo de Nunes (2007) analisou as características de interesses de jovens, quando avaliados com o SDS. Essa pesquisa contou com 289 estudantes da primeira à terceira série do Ensino Médio, sendo $56 \%$ do sexo feminino, com média de idade de 16 anos. A autora analisou a freqüência das tipologias do SDS, tendo verificado que os tipos com escores mais elevados, foram 0 Empreendedor $(28,7 \%)$ e o Social $(21,1 \%)$. Em outra medida, os escores mais baixos foram observados entre os tipos Realista (8\%) e Convencional (9\%). Foram observadas diferenças significativas em função do sexo para os tipos Realista, Social e Convencional, sendo que os homens obtiveram médias mais altas para o Realista e as mulheres, para Social e Convencional. No que diz respeito às variações associadas à idade e série escolar, não foram verificadas diferenças significativas em nenhum dos tipos do SDS.

Em acréscimo, Sartori (2007) avaliou o perfil de preferências profissionais de 131 estudantes de Ensino Médio, de escolas particulares, com idade variando de 15 e 19 anos, média 15,90, sendo 50\% do sexo feminino. A autora utilizou para avaliar os interesses profissionais a Escala de Aconselhamento Profissional (EAP) e o SDS. Os resultados apresentaram correlações significativas entre Ciências Exatas e os tipos Realista $(r=35 ; p<0,05)$, Investigativo $(r=53 ; p<0,01)$ e Convencional $(r=0,19 ; p<0,05)$. A dimensão Artes e Comunicação correlacionou-se com os tipos Artístico $(r=0,64 ; p<0,01)$, Social $(r=0,38$; $p<0,05)$ e Empreendedor $(r=0,23 ; p<0,05)$ e a dimensão Ciências Biológicas e da Saúde com os tipos Investigativo $(r=0,27 ; p<0,05)$ e Social $(r=0,24$; $\mathrm{p}<0,05)$.

Por fim, a dimensão Ciências Humanas e Sociais Aplicadas apresentou correlações com os tipos Investigativo $(r=0,38 ; p<0,05)$, Artístico $(r=0,35$; $p<0,05)$, Social $(r=0,39 ; p<0,01)$ e Empreendedor $(r=0,24 ; p<0,05)$. Ainda, $a$ autora verificou possível diferença de média entre sexos, e encontrou que somente na área de Ciências Biológicas e da Saúde houve significância para o sexo masculino. A autora afirma que o EAP apresentou condições de avaliar preferências profissionais por meio das correlações significativas apresentadas com o SDS, demonstrando que sua utilização em processos de Orientação Profissional permite gerar informações sobre as preferências profissionais. 
Nessa mesma direção, com o objetivo de analisar diferenças de interesse associado a sexo, Martins, Noronha e Godoy (2008) investigaram 107 estudantes do $3^{\circ}$ ano do Ensino Médio de uma escola particular da região Sul do país, participantes de um Programa de Orientação Profissional. A média de idade foi de 16,57 , sendo $44,9 \%$ do sexo masculino e $55,1 \%$ do feminino. As autoras aplicaram o SDS e os achados apresentaram maior interesse masculino nos tipos Realista, Empreendedor e Investigativo, corroborando resultados anteriormente descritos. Por outro lado, as mulheres tiveram médias mais altas nos tipos Artístico e Social e o tipo Convencional, embora não fossem significativamente diferentes. A presente investigação manteve a mesma linha dos estudos relatados, uma vez que objetivou analisar as diferenças de interesses e personalidade em função do sexo e da série escolar.

\section{MÉTODO}

\section{Participantes}

A amostra foi formada por 122 adolescentes do Ensino Médio de uma escola particular do interior paulista. Desses, $44,3 \%$ eram do sexo masculino e $55,7 \%$ do sexo feminino, com média de idade $15,79(D P=8,55)$. No que se refere à distribuição de séries, 27,9\% dos jovens freqüentavam a primeira, 27,9\% freqüentavam a segunda e 44,2\% eram alunos da terceira série do Ensino Médio. Vale destacar que a primeira série é composta, em sua maioria, por alunos de 15 anos de idade $(76,5 \%)$, assim como a segunda $(52,9 \%)$ e a terceira, por alunos de 17 anos de idade (51,9\%).

\section{Instrumentos}

Foram utilizados para a coleta de dados a Escala de Aconselhamento Profissional (EAP) e o Inventário Fatorial de Personalidade (IFP).

\section{Escala de Aconselhamento Profissional (EAP)}

Esse instrumento foi desenvolvido por Noronha, Sisto e Santos (2007) com vistas a auxiliar na identificação das preferências dos participantes por atividades profissionais. A escala é composta de 61 itens, com formato Likert e as respostas devem variar de freqüentemente (5) a nunca (1), de acordo com o interesse do avaliando em desenvolver cada atividade. 
Dentre os estudos psicométricos, destaca-se o de validade de construto que, por meio da análise fatorial, chegou a uma solução composta por sete fatores, a saber: ciências exatas, artes e comunicação, ciências biológicas e da saúde, ciências agrárias e ambientais, atividades burocráticas, ciências humanas e sociais aplicadas, entretenimento, com índices de saturação dos itens nos fatores superiores a 0,30 e variância explicada de 57,31\%.

No que se refere à precisão do instrumento, houve a verificação por meio da consistência interna, a qual avalia a uniformidade do instrumento com relação aos seus itens. Dentre as formas para estudar a consistência interna, tratando-se da Teoria Clássica dos Testes, escolheu-se o coeficiente alfa de Cronbach e a correlação de duas metades, cujos valores de alfa ficaram entre 0,79 e 0,94 e os de Spearman-Brown e Guttman entre 0,75-0,91, considerando-se assim que as diversas dimensões do instrumento são precisas.

Inventário Fatorial de Personalidade (IFP)

O IFP foi desenvolvido por Pasquali, Azevedo e Ghesti em 1997, fundamentado nos traços de personalidade de Edwards Personal Preference Schedule (EPPS), o inventário avalia no total 15 fatores, em 155 itens, sendo 135 itens referentes aos traços de personalidade, além das duas escalas que avaliam a desejabilidade social e a validade das respostas dos sujeitos ao instrumento. A escala é tipo Likert, composta de 7 pontos que correspondem progressivamente de ' $1=$ Nada característico' até '7=Totalmente característico'.

Com o objetivo de se aferir a validade de construto, realizou-se análise fatorial confirmatória, dentre os 15 fatores propostos pela teoria do teste, 11 explicaram cerca de $40 \%$ da variância de seus respectivos itens. Estes fatores apresentaram, em sua maioria, carga fatorial igual ou superior a 0,30. Destes, 6 possuíam uma consistência interna entre 0,78 a 0,84 e o restante com o mesmo índice superior a 0,75 , o que justificou a validade do instrumento.

\section{Procedimento}

Após aprovação do Comitê de Ética em Pesquisa de uma instituição de ensino superior, e mediante a assinatura do Termo de Consentimento Livre e Esclarecido pelos respectivos responsáveis, os participantes responderam aos testes coletivamente em sala de aula, em uma única sessão, em horário previamente agendado com a instituição. As turmas foram divididas em primeiro, 
segundo e terceiro anos e o tempo de aplicação foi de aproximadamente 1 hora. Aplicou-se primeiramente o EAP e em seguida o IFP.

\section{RESULTADOS E DISCUSSÃO}

Os dois testes foram avaliados quanto à existência de possíveis diferenças de média em função das variáveis sexo e série escolar, sendo para tanto, utilizados o teste $t$ de Student e a análise de variância (ANOVA). A Tabela 1 apresenta a média e o desvio padrão para cada sexo nas sete dimensões do EAP. $O$ teste $t$ de Student revelou que houve diferença de média entre os sexos nas dimensões Ciências Exatas, Artes e Comunicação, Ciências Biológicas e da Saúde, Ciências Agrárias e Ambientais, Ciências Humanas e Sociais Aplicadas e Entretenimento.

No que tange à dimensão Ciências Exatas $[\mathrm{t}(122)=4,171 ; \mathrm{p}=0,000]$, o sexo masculino apresentou médias maiores. Dados semelhantes foram obtidos por Martins et al (2008), Nunes (2007) e Osborn e Reardon (2006), de modo que os autores encontraram preferência masculina no tipo Realista que, de acordo com Holland (1997), as características nessa tipologia são a boa habilidade matemática, a preferência por problemas concretos, trabalhos em ambientes abertos como serviços agrícolas e engenharia.

Tabela 1.

Média e desvio-padrão nas dimensões do EAP em função do sexo (Amostra masculina $=54$; Amostra feminina $=68$ ).

\begin{tabular}{|c|c|c|c|c|c|}
\hline Dimensões do EAP & $\mathrm{t}$ & $p$ & Sexo & Média & $D P$ \\
\hline \multirow{2}{*}{ Ciências Exatas } & \multirow{2}{*}{4,171} & \multirow{2}{*}{0,000} & Masculino & 38,15 & 13,626 \\
\hline & & & Feminino & 29,10 & 10,326 \\
\hline \multirow{2}{*}{ Artes e Comunicação } & \multirow{2}{*}{$-3,947$} & \multirow{2}{*}{0,000} & Masculino & 30,57 & 10,717 \\
\hline & & & Feminino & 38,84 & 12,063 \\
\hline \multirow{2}{*}{$\begin{array}{l}\text { Ciências Biológicas e da } \\
\text { Saúde }\end{array}$} & \multirow{2}{*}{$-2,757$} & \multirow{2}{*}{0,007} & Masculino & 17,69 & 7,397 \\
\hline & & & Feminino & 21,79 & 8,744 \\
\hline \multirow{2}{*}{$\begin{array}{l}\text { Ciências Agrárias e } \\
\text { Ambientais }\end{array}$} & \multirow{2}{*}{$-4,475$} & \multirow{2}{*}{0,000} & Masculino & 28,37 & 10,690 \\
\hline & & & Feminino & 36,85 & 10,162 \\
\hline \multirow{2}{*}{ Atividades Burocráticas } & \multirow{2}{*}{1,187} & \multirow{2}{*}{0,132} & Masculino & 35,09 & 11,088 \\
\hline & & & Feminino & 33,22 & 8,936 \\
\hline \multirow{2}{*}{$\begin{array}{l}\text { Ciências Humanas e Sociais } \\
\text { Aplicadas }\end{array}$} & \multirow{2}{*}{$-3,295$} & \multirow{2}{*}{0,001} & Masculino & 21,04 & 6,718 \\
\hline & & & Feminino & 25,10 & 6,811 \\
\hline \multirow{2}{*}{ Entretenimento } & \multirow{2}{*}{$-3,419$} & \multirow{2}{*}{0,001} & Masculino & 13,13 & 6,553 \\
\hline & & & Feminino & 17,32 & 6,864 \\
\hline
\end{tabular}


Por outro lado, os achados de Osborn e Reardon (2006) e Williams e Subich (2006) apresentaram preferência masculina no tipo Realista, o que contrapõe o resultado da dimensão Ciências Agrárias e Ambientais $[\mathrm{t}(122)=-4,475$; $p=0,000]$, no qual as mulheres apresentaram médias mais elevadas. Vale destacar que a população avaliada nestas pesquisas é americana, enquanto que no presente estudo, foram investigados alunos brasileiros, o que pode ter influenciado na magnitude das preferências encontradas devido diferenças culturais.

Sob essa perspectiva, houve significância também em relação à dimensão Artes e Comunicação $[t(122)=-3,947 ; p=0,000]$, Ciências Biológicas e da Saúde $[t(122)=-2,757 ; \quad p=0,007]$, Ciências Humanas e Sociais Aplicadas $[t(122)=-3,295 ; p=0,001]$ e Entretenimento $[t(122)=-3,419 ; p=0,001]$ com médias maiores para o sexo feminino. Os resultados são parcialmente concordantes com os achados internacionais de Osborn e Reardon (2006) e nacionais de Martins et al (2008) que, ao investigarem diferenças de sexo no SDS, as mulheres apresentaram médias maiores para os tipos Social e Artístico (SDS), cujas preferências coincidem com as características das dimensões Artes e Comunicação e Ciências Humanas Sociais Aplicadas (EAP). Ainda, esses dados reforçam o estudo de Nunes (2007) e Reardon et al (2005) no que diz respeito aos escores elevados no tipo Social para mulheres, assim como o estudo de Almeida et al (2006), no qual os autores encontraram preferência feminina nas áreas das Ciências Sociais, que, na EAP, estão mais especificadas na dimensão Ciências Humanas Sociais Aplicadas.

A investigação de Santos e Melo-Silva (2005) sobre as preferências por cursos de graduação associadas ao sexo corrobora os escores obtidos nas dimensões Ciências Biológicas e da Saúde e Ciências Humanas e Sociais Aplicadas, uma vez que as mulheres estiveram mais presentes em cursos como enfermagem, fonoaudiologia, terapia ocupacional, nutrição, farmácia, psicologia, pedagogia, fisioterapia, os quais envolvem cuidado nos aspectos físico e psicológico, além do caráter assistencial. A pesquisa de Almeida et al (2006) também condiz o presente achado, no sentido da maior participação feminina nas Ciências Naturais. Em outra medida, os achados de Sartori (2007) apresentaram pontuação significativa para o sexo masculino na dimensão Ciências Biológicas e da Saúde. No entanto, supõe-se que as discrepâncias entre 
os resultados deste caso, estejam relacionadas a características contextuais específicas das amostras.

A dimensão Atividades Burocráticas não apresentou diferenças significativas com relação ao sexo. Esse resultado não se mostra pertinente com os estudos de Nunes (2007), Reardon et al (2005) e Carless (1999), uma vez que os autores encontraram preferência feminina no tipo Convencional, cujas características são similares à dimensão supracitada. Nessa tipologia, Holland (1997) refere-se a pessoas que gostam de manipular e analisar dados, atividades sistemáticas, passivas e organizadas, conferindo muita importância para organização de materiais e de ambientes. Nesse particular, considerando as informações presentemente obtidas, sugerem-se novos estudos que busquem comparar 0 atual resultado.

Quanto à dimensão Entretenimento, o conteúdo dos itens versa sobre assuntos associados à moda, publicidade e gerenciamento de atividades de lazer (Noronha, Sisto \& Santos, 2007). No entanto, destacam-se características associadas à sensibilidade artística, assim como o contato social como forma de trabalho. Esse resultado está em consonância com os achados de Martins et al (2008), Osborn e Reardon (2006) e Carless (1999), nos quais as mulheres apresentaram médias maiores no tipo Artístico.

De maneira semelhante, observaram-se diferenças significativas em função do sexo em alguns fatores do IFP, conforme observado na Tabela 2. No que se refere aos fatores Assistência $[\mathrm{t}(122)=-3,275 ; \mathrm{p}=0,002]$, Intracepção $[\mathrm{t}(122)=-$ 2,870; $\mathrm{p}=0,005]$, Afago $[\mathrm{t}(122)=-3,984 ; \mathrm{p}<0,000]$, Afiliação $[\mathrm{t}(122)=-2,962 ;$ $\mathrm{p}=0,004]$, Denegação $[\mathrm{t}(122)=-1,942 ; \mathrm{p}=0,055]$, Ordem $[\mathrm{t}(122)=-1,942$; $\mathrm{p}=0,054]$ e Mudança $[\mathrm{t}(122)=-3,634 ; \mathrm{p}<0,000]$, o sexo feminino apresentou médias maiores. Sob essa perspectiva, de acordo com os fatores definidos no Manual do IFP por Pasquali et al (1997), pode-se inferir que, nessa amostra específica, as mulheres tendem a apresentar características voltadas para sentimentos de compaixão, ternura, fantasia, imaginação, proteção, apoio, confiança, amor, culpa, fracasso, organização, equilíbrio, além do desejo de obter coisas novas, mudar de hábitos e lugares. 
Tabela 2.

Média e desvio-padrão nos fatores do IFP em função do sexo (Amostra masculina $=54$; Amostra feminina $=68$ )

\begin{tabular}{|c|c|c|c|c|c|}
\hline Fatores do IFP & $t$ & $p$ & Sexo & Média & $D P$ \\
\hline \multirow{2}{*}{ Assistência } & \multirow{2}{*}{$-3,275$} & \multirow{2}{*}{0,002} & Masculino & 44,37 & 9,110 \\
\hline & & & Feminino & 49,12 & 7,102 \\
\hline \multirow{2}{*}{ Intracepção } & \multirow{2}{*}{$-2,870$} & \multirow{2}{*}{0,005} & Masculino & 41,02 & 10,618 \\
\hline & & & Feminino & 45,96 & 8,387 \\
\hline \multirow{2}{*}{ Afago } & \multirow{2}{*}{$-3,984$} & \multirow{2}{*}{0,000} & Masculino & 37,54 & 11,060 \\
\hline & & & Feminino & 45,63 & 6,740 \\
\hline \multirow{2}{*}{ Deferência } & \multirow{2}{*}{1,580} & \multirow{2}{*}{0,073} & Masculino & 43,46 & 8,650 \\
\hline & & & Feminino & 45,72 & 7,592 \\
\hline \multirow{2}{*}{ Afiliação } & \multirow{2}{*}{$-2,962$} & \multirow{2}{*}{0,004} & Masculino & 51,06 & 8,947 \\
\hline & & & Feminino & 55,25 & 6,692 \\
\hline \multirow{2}{*}{ Dominância } & \multirow{2}{*}{2,213} & \multirow{2}{*}{0,029} & Masculino & 40,46 & 9,747 \\
\hline & & & Feminino & 36,60 & 9,422 \\
\hline \multirow{2}{*}{ Denegação } & \multirow{2}{*}{$-1,942$} & \multirow{2}{*}{0,055} & Masculino & 34,22 & 8,135 \\
\hline & & & Feminino & 36,97 & 7,461 \\
\hline \multirow{2}{*}{ Desempenho } & \multirow{2}{*}{2,708} & \multirow{2}{*}{0,008} & Masculino & 54,02 & 5,910 \\
\hline & & & Feminino & 50,81 & 6,935 \\
\hline \multirow{2}{*}{ Exibição } & \multirow{2}{*}{1,237} & \multirow{2}{*}{0,121} & Masculino & 42,87 & 10,161 \\
\hline & & & Feminino & 41,12 & 9,148 \\
\hline \multirow{2}{*}{ Agressão } & \multirow{2}{*}{$-3,672$} & \multirow{2}{*}{0,000} & Masculino & 41,41 & 10,478 \\
\hline & & & Feminino & 34,96 & 8,918 \\
\hline \multirow{2}{*}{ Ordem } & & & Masculino & 42,96 & 10,878 \\
\hline & & & Feminino & 46,40 & 8,658 \\
\hline & & & Masculino & 45,70 & 8,364 \\
\hline Persistencia & & & Feminino & 43,32 & 8,558 \\
\hline Mudanca & & & Masculino & 43,69 & 9,542 \\
\hline Mudança & & & Feminino & 49,57 & 8,339 \\
\hline Auton & & & Masculino & 49,78 & 6,488 \\
\hline Autonomia & & & Feminino & 46,40 & 6,600 \\
\hline & & & Masculino & 50,59 & 7,810 \\
\hline Heteross & -5 & & Feminino & 42,76 & 7,234 \\
\hline
\end{tabular}

Por outro lado, o sexo masculino obteve médias mais elevadas nos fatores Dominância $[t(122)=2,213 ; p=0,029]$, Desempenho $[t(122)=2,708 ; p=0,008]$, Agressão $[t(122)=-3,672 ; p=0,000]$, Autonomia $[t(122)=2,831 ; p=0,005]$ e Heterossexualidade $[\mathrm{t}(122)=-5,731 ; \mathrm{p}=0,000]$. Neste caso, ainda de acordo com a definição dos fatores de Pasquali et al (1997), os homens se destacaram nas características de persuasão, sedução, comando, domínio, manipulação, independência e liberdade e desejo de manter relacionamentos românticos e sexuais com pessoas do sexo oposto. Os fatores Deferência, Exibição e Persistência não apresentaram diferenças significativas com relação ao sexo. 
Com objetivo de verificar possíveis diferenças de média dos interesses e das características de personalidade em razão das séries, realizou-se análise de variância (ANOVA), cujos resultados são apresentados na Tabela 3 e na Tabela 4.

No que se refere aos interesses, não foram encontradas diferenças significativas entre os primeiro, segundo e terceiro anos do Ensino Médio. No entanto, acredita-se que seja possível não existir diferenciação entre as séries no que se refere às preferências profissionais, mesmo supondo-se que, em outra medida, estudantes das séries mais avançadas possam mostrar algumas de suas escolhas mais definidas (Noronha et al, 2007).

Esses resultados são congruentes com os estudos de Sartori (2007) e Nunes (2007) que, ao aplicarem o SDS em alunos do Ensino Médio, não encontraram diferenças significativas de interesses em função da idade ou série escolar. Portanto, não se pode afirmar que níveis diferentes de escolaridade influenciam no interesse por uma profissão.

Tabela 3. Análise de variância do EAP em função da série escolar

\begin{tabular}{lcc}
\hline \multicolumn{1}{c}{ Dimensões do EAP } & $F$ & $p$ \\
\hline Ciências Exatas & 2,407 & 0,094 \\
\hline Artes e Comunicação & 0,863 & 0,425 \\
\hline Ciências Biológicas e da Saúde & 2,396 & 0,095 \\
\hline Ciências Agrárias e Ambientais & 0,627 & 0,536 \\
Atividades Burocráticas & 1,951 & 0,147 \\
Ciências Humanas e Sociais & 0,190 & 0,827 \\
Aplicadas & 1,924 & 0,151 \\
\hline Entretenimento & & \\
\hline
\end{tabular}

A Tabela 4 apresenta os resultados encontrados por meio da ANOVA do IFP em função da série escolar. De um modo geral, assim como ocorreu na análise dos interesses, não foram encontradas diferenças significativas no que tange às características de personalidade, com exceção do fator Exibição. 
Tabela 4. Análise de variância do IFP em função da série escolar

\begin{tabular}{lcc}
\hline \multicolumn{1}{c}{ Fatores do IFP } & $F$ & $p$ \\
\hline Assistência & 0,052 & 0,949 \\
\hline Intracepção & 0,200 & 0,819 \\
\hline Afago & 0,210 & 0,810 \\
\hline Deferência & 2,063 & 0,132 \\
\hline Afiliação & 0,052 & 0,950 \\
\hline Dominância & 2,153 & 0,121 \\
\hline Denegação & 0,194 & 0,824 \\
\hline Desempenho & 0,842 & 0,434 \\
\hline Exibição & 3,486 & $\mathbf{0 , 0 3 4}$ \\
\hline Agressão & 0,682 & 0,508 \\
\hline Ordem & 1,878 & 0,157 \\
\hline Persistência & 0,058 & 0,944 \\
\hline Mudança & 0,539 & 0,585 \\
\hline Autonomia & 2,001 & 0,140 \\
\hline Heterossexualidade & 0,023 & 0,977 \\
\hline
\end{tabular}

Os achados estão em consonância com o estudo de Balbinotti et al (2006) que ao avaliarem os interesses profissionais de soldados do Exército Brasileiro por meio da aplicação do SDS, a variável faixa etária não determinou diferenças nos níveis de interesses profissionais quanto às suas personalidades vocacionais. Esse dado, contudo, deve ser analisado com cautela, uma vez que a faixa etária consultada nas duas amostras se diferenciava, além das ocupações dos dois grupos serem diferentes (estudantes do Ensino Médio e profissionais - soldados, que já passaram pelo processo de escolha de uma profissão).

\section{CONSIDERAÇÕES FINAIS}

O presente artigo teve como objetivo analisar as diferenças de interesses e personalidade em função do sexo e da série escolar. Os resultados mostraram-se em consonância com as pesquisas realizadas e apresentadas na fundamentação desse estudo, como Martins et al (2008), Nunes (2007), Osborn e Reardon (2006), Santos e Melo-Silva (2005), de modo a confirmar a existência de estereótipos associados a gênero no que se refere às preferências profissionais e às características de personalidade.

No que tange às diferenças de interesses e características de personalidade em razão da série escolar, de um modo geral, não foram encontradas diferenças 
significativas entre o primeiro, segundo e terceiros anos do Ensino Médio. No entanto, outras faixas etárias poderão ser investigadas com o EAP, uma vez que se acredita que o processo de desenvolvimento vocacional é contínuo e que as escolhas profissionais podem ser revisadas em diferentes momentos da vida. Recomenda-se que os estudos sobre Orientação Profissional continuem em foco, com intuito de contribuir com as reflexões a respeito dos construtos envolvidos no processo de escolha.

Desta forma, conclui-se que a OP como área das ciências humanas precisa dispor cada vez mais de conhecimento teórico e prático no contexto social, político e econômico. A avaliação dos instrumentos, dos processos e dos resultados, assim como as estratégias utilizadas nos procedimentos de intervenção, tal como afirmado por Melo-Silva e cols. (2003), requerem pesquisas contínuas e a divulgação delas no âmbito científico e, principalmente, no prático. Por tanto, muitos outros estudos serão ainda necessários, tomandose como referência o cenário nacional, que se encontra ainda em desenvolvimento e expansão na área de Orientação Profissional.

\section{REFERÊNCIAS}

Almeida, L. S., Guisande, M. A., Soares, A. P., \& Saavedra, L. (2006). Acesso e sucesso no ensino superior em Portugal: Questões de género, origem sóciocultural e percurso académico dos alunos. Psicologia: Reflexão e Crítica, 19(3), 507-514.

Balbinotti, M. A. A., Valentini, F. \& Cândido, M. O. (2006). Níveis de interesses profissionais em soldados do exército brasileiro. Revista Brasileira de Orientação Profissional, 7(1), 23-36.

Bohoslavsky, R. (1993). Orientação vocacional: A estratégia clínica. São Paulo: Martins Fontes.

Carless, S. (1999). Career Assessment: Holland's Vocational Interests, Personality Characteristics, and Abilities. Assessment Journal of Career, $7(2), 125-144$.

Duarte, M. E. (2008). A Avaliação Psicológica na Intervenção Vocacional: Princípios, Técnicas e Instrumentos. In M. C. Taveira \& J. T. Silva (Eds.), Psicologia Vocacional Perspectivas para a Intervenção. (pp. 139-157). Coimbra: Imprensa da Universidade de Coimbra. 
Holland, J. L. (1963). Explorations of a theory of vocational choice and achievement: II. A four-year prediction study. Psychological Reports, 12, 547-594.

Holland, J. L. (1997). Making vocational choices: A theory of vocational personalities and work environments ( $2^{\mathrm{a}}$ ed.). Odessa, FL: Psychological Assessment Resources.

Leitão, L. M., \& Miguel, J. P. (2004). Avaliação dos interesses. In L. M. Leitão (Eds.), Avaliação Psicológica em Orientação Escolar e Profissional. (pp. 179262). Coimbra: Quarteto.

Martins, D. F., Noronha, A. P. P. \& Godoy, S. (2008). Análise dos interesses de estudantes do ensino médio de um programa de orientação profissional. In A. P. Noronha, C. Machado, L. Almeida, M. Gonçalves, S. Martins \& V. Ramalho. (Eds.), Actas da XIII Conferência Internacional - Avaliação Psicológica: Formas e Contextos. Braga: Psiquilíbrios.

Mattiazzi, B. (1977). A natureza dos interesses e a orientação vocacional. Petrópolis: Vozes.

Melo-Silva, L. L., Noce, M. A. \& Andrade, P. P. (2003). Interesses em adolescentes que procuram orientação profissional. Psic, 4(2), 06-17.

Noronha, A. P. P., Sisto, F. F., \& Santos, A. A. A. (2007). Escala de Aconselhamento Profissional - EAP. Manual de Aplicação. São Paulo: Vetor Editora Psico-Pedagógica Ltda.

Nunes, M. F. O. (2007). Escala de Fontes de Eficácia Percebida: Aplicação com jovens em escolha profissional. Dissertação de Mestrado, Programa de PósGraduação Stricto Sensu em Psicologia, Universidade São Francisco, Itatiba. Nunes, M. F. O., \& Noronha, A. P. P. (2009). Relações entre interesses, personalidade e habilidades cognitivas. Psico-USF, 14(2), 131-141.

Osborn, D. S., \& Reardon, R. C. (2006). Using the Self-Directed Search: Career explorer with high-risk middle school students. Career Development Quarterly, 54(3), 269-273.

Pasquali, L., Azevedo, M. M., \& Ghesti, I. (1997). Inventário Fatorial de Personalidade - IFP Manual Técnico de Aplicação. São Paulo: Casa do Psicólogo. 
Reardon, R. C., Bullock, E. E., \& Meyer, K. E. (2005). A Holland Perspective on the U.S. Workforce from 1960 to 2000 - Technical Report No. 45. Tallahassee, FL: Center for the Study of Technology in Counseling and Career Development- The Florida State University.

Santos, S. G., \& Melo-Silva, L. L. (2005). Questão de Gênero e Escolha Profissional. Em M. C. P. Lassance (Ed.). Intervenção e Compromisso Social. (pp. 263-290). São Paulo: Vetor Editora Psico-Pedagógica Ltda.

Sartori, F. A. (2007). Estudo Correlacional entre a Escala de Aconselhamento Profissional (EAP) e o Self-Directed Search Career Explorer (SDS). Dissertação de Mestrado, Programa de Pós-Graduação Stricto Sensu em Psicologia, Universidade São Francisco, Itatiba.

Savickas, M. L. (1999). The psychology of interests. Em M. L. Savickas \& A. R. Spokane (Eds.), Vocational interests. Meanings, Measurement and Counseling Use. (pp. 19-56). Palo Alto, CA: Davies-Black.

Williams, M. C. \& Subich, C. M. (2006). The gendered nature of career related learning experiences: A social cognitive career theory perspective. Journal of Vocational Behavior, 69, 262-275.

Contato: silviagodoy04@yahoo.com.br

Recebido em: 29/09/2010

Revisado em: 04/11/2010

Aceito em: 03/12/2010 